ROCKY MOUNTAIN

JOURNAL OF MATHEMATICS

Volume 24, Number 1, Winter 1994

\title{
AN EPIDEMIOLOGICAL MODEL WITH POPULATION SIZE DEPENDENT INCIDENCE
}

\author{
JINSHI ZHOU
}

Dedicated to Paul Waltman on the occasion of his 60th birthday

\begin{abstract}
An SIS epidemiological model with a general population-size dependent disease incidence, reduced fertility and vertical transmission is analyzed. Complete global stability analyses and explicit threshold parameters are given. The fractions in the disease states usually approach a disease-free or endemic equilibrium while the population size grows to infinity, decays to zero or approaches a finite size. In one case there is a center. The persistence of the disease and diseaserelated deaths affects the behavior of the population size.
\end{abstract}

1. Introduction. Consider the spread of an infectious disease in a host population with exponential births and deaths defined as follows. Let $N(t)$ be the total population size as a function of time $t, b$ be the natural birth rate constant, and $d$ be the natural death rate constant. Then, assuming that the natural births and the natural deaths are proportional to the total population size, we have

$$
N^{\prime}(t)=(b-d) N, \quad N(0)=N_{0}
$$

as the initial value problem. The net growth rate is therefore $r=b-d$ so that the population size $N(t)$ grows exponentially if $r>0$, is constant if $r=0$ and decays exponentially if $r<0$.

Assume that the rate at which new individuals become infected (the incidence rate) is proportional to number $Y$ of infectives, times the population-size dependent contact rate $\lambda(N)$, times the probability of contacting a susceptible individual, $X / N$, where $X$ is the number of susceptible individuals. Thus the incidence is $\lambda(N) Y X / N$. The contact rate $\lambda(N)$ is the daily average number of contacts of an infective which are sufficient for transmission if the person contacted is susceptible

Received by the editors on March 9, 1993.

Key words. Epidemiological model, global stability, nonlinear incidence rate, thresholds, varying population size.

Copyright (c)1994 Rocky Mountain Mathematics Consortium 
so that $\lambda(N) Y$ is the daily total number of adequate contacts of all infectives. If $\lambda(N)=\beta N$, this is the classical mass action incidence which is considered by many authors such as Anderson and May [1], Mena-Lorca and Hethcote [13]. If $\lambda(N)=\beta$, this is the standard incidence which is discussed by Busenberg and Van den Driessche [7], Busenberg and Hadeler [6], Gao and Hethcote [10], Mena-Lorca and Hethcote [13].

The above two choices for $\lambda(N)$ are rather specific and are only two extreme cases. When Anderson [2] fit $\lambda(N)=\beta N^{a}$ to several communities and several childhood diseases, he found best fits for $a$ between 0.03 and 0.07. Andreason $[3]$ reported that $\lambda(N)=\beta N^{1 / 2}$ seems to give the best fit to some data on rabbit myxomatosis. However, due to the limitation of the availability of data and the constant size of the communities that are measured, these estimates do not necessarily reflect how the contact rate changes in communities with varying population size. Other choices for $\lambda(N)$ are $\lambda(N)=N /\left(K_{1}+K_{2} N\right)$ for venereal diseases (which is an analogy to Holling's type II functional response [12]), and $\lambda(N)=N /\left(1+K_{1}+\sqrt{1+2 K_{2} N}\right)$ for short time social complexes [11]. These two forms of $\lambda(N)$ approximate the form of the classical mass action incidence for small population size and the standard incidence for large population size and interpolate in between. Our goal is to consider the general form $\lambda(N)$ which can include all of the choices above.

The population-size dependent contact function $\lambda(N)$ has become more important recently, particularly in models for the spread of HIV (the virus related to AIDS); see Castillo-Chavez et al., [8, 9], and Thieme and Castillo-Chavez [17]. General population-size dependent contact functions have also been considered by Brauer $[4,5]$; Pugliese $[\mathbf{1 5}, \mathbf{1 6}]$; and Thieme $[\mathbf{1 8}, \mathbf{1 9}]$.

In this paper we consider the effects of a nonlinear contact rate in a model where the infectious disease can affect the host population size. The epidemic model itself is of SIS type which includes vital dynamics influenced by the disease and vertical transmission. Age structure and other delays are neglected, so that the model is a nonlinear system of ordinary differential equations. Threshold conditions are obtained which determine the global behavior of the model, that is, whether the disease dies out or remains endemic and whether the population size decays to zero, remains finite or grows exponentially. For certain 
parameter values, there is an endemic center so that the disease and population are oscillatory.

2. The SIS model. The host population $N(t)$ is divided into susceptibles, $X(t)$, and infectives, $Y(t)$, which change with time $t$. The fertility of susceptibles is $b$, that of infectives is $b \varepsilon$, with $\varepsilon \in[0,1]$ so $\varepsilon$ is the fertility reduction factor due to infection. Assume that the newborns of infectives are susceptibles with probability $\rho$ in $[0,1]$ and infectives with probability $(1-\rho)$ so that $1-\rho$ is the probability of vertical transmission from a mother to her newborn baby before, during, or just after birth. The natural death rate constant is $d$, the disease-related death rate constant is $\alpha$ and the recovery rate constant is $\gamma$. Here we follow Mena-Lorca and Hethcote's [13] convention that Roman letters are used for demographic parameters and Greek letters are used for epidemiologic parameters.

The transfer diagram for the SIS model is

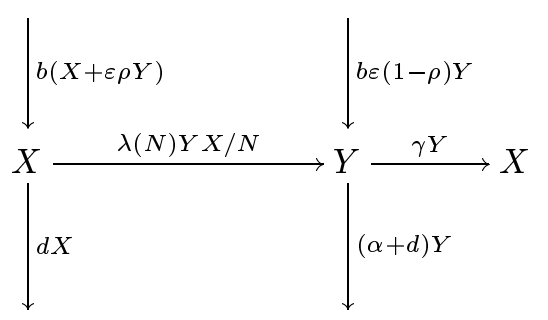

The differential equations for this model are:

$$
\begin{aligned}
X^{\prime}(t) & =b(X+\rho \varepsilon Y)-\frac{\lambda(N)}{N} X Y-d X+\gamma Y \\
Y^{\prime}(t) & =\frac{\lambda(N)}{N} X Y+b \varepsilon(1-\rho) Y-(\gamma+\alpha+d) Y \\
N^{\prime}(t) & =(b-d) N-(\alpha+b(1-\varepsilon)) Y
\end{aligned}
$$

where one of the equations is redundant since $N=X+Y$. For biological significance, we assume that all of the parameters are nonnegative. In view of several forms of $\lambda(N)$ given above, we make the following assumptions:

i) $\lambda(N)$ is continuous for $N \geq 0$ and is continuously differentiable for $N>0$. 
ii) $\lambda(N)>0$ if $N>0$.

iii) $\lambda(N)$ is a nondecreasing function of $N$.

It is easy to check that the first quadrant in $X Y$ space is positively invariant under the solution flow of (2.1) and unique solutions exist for $t \geq 0$ so that this model is mathematically and epidemiologically well-posed.

The net growth rate constant in a disease free population is $r=$ $b-d$. In the absence of disease, the population size $N(t)$ declines exponentially to zero if $r<0$, remains constant if $r=0$ and grows exponentially if $r>0$. If the disease is present, the population still declines to zero if $r<0$. For $r>0$ the population can go to zero, remain finite or grow exponentially and the disease can die out or persist depending on the values of several threshold quantities.

It is convenient to reformulate the model (2.1) in terms of the fraction $I=Y / N$ which is infectious. The susceptible fraction $S=X / N$ satisfies $S=1-I$. The last two differential equations in (2.1) become

$$
\begin{aligned}
I^{\prime}(t) & =\left(\lambda(N)-\left(\alpha_{1}+\gamma+b \varepsilon \rho\right)\right) I-\left(\lambda(N)-\alpha_{1}\right) I^{2} \\
N^{\prime}(t) & =N\left(r-\alpha_{1} I\right)
\end{aligned}
$$

where $\alpha_{1}=\alpha+b(1-\varepsilon)$ corresponds to the deaths and reduced births due to the disease. The relevant region is

$$
D=\{(I, N) \mid 0 \leq I \leq 1, N \geq 0\}
$$

which is positively invariant. For any initial data in $D,(2.2)$ has a unique global solution in $D$.

Note that the combination of the horizontal and vertical contact rate is $\lambda(N)+b \varepsilon(1-\rho)$. In model $(2.1)$ the transfer rate $-(\gamma+\alpha+$ d) $Y$ corresponds to an exponentially distributed waiting time in the infectious population $Y$ with the mean waiting time in $Y$ given by $1 /(\gamma+\alpha+d)$. Thus the contact number defined by

$$
\sigma_{N}=\frac{\lambda(N)+b \varepsilon(1-\rho)}{\alpha+d+\gamma}
$$

is the average number of adequate contacts at population size $N$ of an infective during the average period in the infectious population 
$Y$. In model $(2.2)$ the transfer rate $-(\gamma+\alpha+b) I$ corresponds to an exponentially distributed waiting time in the infectious fraction $I$ with the mean waiting time in $I$ given by $1 /(\gamma+\alpha+b)$. Note that the mean waiting time in $Y$ is generally not the same as the mean waiting time in $I$ since the population size is changing. The modified contact number defined by

$$
\theta_{N}=\frac{\lambda(N)+b \varepsilon(1-\rho)}{\alpha+b+\gamma}
$$

is the average number of adequate contacts at population size $N$ of an infective during the average period in the infectious fraction $I$. These contact number definitions generalized those in Mena-Lorca and Hethcote [13], Gao and Hethcote [10].

The growth threshold at population size $N$ is defined by

$$
\psi_{N}=\frac{r}{\alpha_{1}}+\frac{|\alpha+d+\gamma-b \varepsilon(1-\rho)|}{\lambda(N)} .
$$

This threshold reflects the relation between the natural net growth rate $r$ and the effects of the disease such as disease-related deaths and horizontal and vertical transmission. The threshold $\psi_{N}$ governs the actual growth of the host population. This threshold $\psi_{N}$ is not a direct generalization of the net growth threshold $\phi$ used in $[\mathbf{1 0}, \mathbf{1 3}]$, but it is closely related and serves the same purpose.

The limiting values of the threshold quantities $\theta_{N}, \sigma_{N}, \psi_{N}$ at both small population size and large population size, i.e., $\theta_{0}, \sigma_{0}, \psi_{0}, \theta_{\infty}, \sigma_{\infty}$, $\psi_{\infty}$, are important in the analysis below. These values are connecting to the limiting values $\lambda(0)$ and $\lambda(\infty)$.

In later sections we use the following result many times.

Lemma 2.1. Consider the solution $u(t)$ of the initial value problem

$$
u^{\prime}(t)=a(t) u-c(t) u^{2}, \quad u(0)=u_{0}>0
$$

where $a(t), c(t)$ are continuous for $t \geq 0$.

(i) If there exists a constant $\delta>0$ such that $a(t) \leq-\delta$ for large $t$, and $c(t) \geq 0$ for large $t$, then $\lim _{t \rightarrow \infty} u(t)=0$. 
(ii) If $\lim _{t \rightarrow \infty} a(t)=0$ and there exists a constant $\delta>0$ such that $c(t) \geq \delta$ for large $t$, then $\lim _{t \rightarrow \infty} u(t)=0$.

(iii) If $\lim _{t \rightarrow \infty} a(t)>0$ and $\lim _{t \rightarrow \infty} c(t)>0$, then

$$
\lim _{t \rightarrow \infty} u(t)=\frac{\lim _{t \rightarrow \infty} a(t)}{\lim _{t \rightarrow \infty} c(t)} .
$$

Proof. Note that solutions of (2.7) remain positive.

(i) Since $u^{\prime}(t) \leq-\delta u$ for large $t$, we must have $\lim _{t \rightarrow \infty} u(t)=0$.

(ii) Let $w=1 / u$, then $w^{\prime}(t)=-a(t) w+c(t)$. For arbitrary $\nu>0$, choose $t_{0}>0$ such that $c(t) \geq \delta$ and $|a(t)|<\nu$ for $t \geq t_{0}$. Then

$$
w^{\prime}=-a(t) w+c(t) \geq-\nu w+\delta .
$$

Integrating this inequality from $t_{0}$ to $t$, we get

$$
w(t) \geq w\left(t_{0}\right) e^{\nu\left(t_{0}-t\right)}+\frac{\delta}{\nu}\left(1-e^{\nu\left(t_{0}-t\right)}\right) \geq \frac{\delta}{\nu}\left(1-e^{\nu\left(t_{0}-t\right)}\right)
$$

Let $t_{1}=(\log 2) / \nu+t_{0}$. Then, for $t \geq t_{1}$, we have $e^{\nu\left(t_{0}-t\right)} \leq 1 / 2$ and

$$
w(t) \geq \frac{\delta}{2 \nu} \quad \text { for } t \geq t_{1} .
$$

Thus, $u \leq 2 \nu / \delta$ for $t \geq t_{1}$. Since $\delta$ is fixed and $\nu$ is arbitrary we obtain that $u(t) \rightarrow 0$ as $t \rightarrow \infty$.

(iii) The change of $u=1 / w$ leads to the linear differential equation $w^{\prime}(t)=-a(t) w+c(t)$ whose solution is

$$
w(t)=\frac{w_{0}+\int_{0}^{t} c(v) \exp \left(\int_{0}^{v} a(s) d s\right) d v}{\exp \left(\int_{0}^{t} a(s) d s\right)} .
$$

There is a $t_{1}>0$ such that $a(t)>a(\infty) / 2$ for $t \geq t_{1}$ and

$$
\exp \left(\int_{0}^{t} a(s) d s\right) \geq \exp \left(\int_{0}^{t_{1}} a(s) d s+a(\infty) \frac{t-t_{1}}{2}\right) .
$$


Since both the numerator and the denominator of (2.8) approach infinity as $t$ approaches infinity, we apply L'Hopital's rule to get

$$
\lim _{t \rightarrow \infty} w(t)=\frac{\lim _{t \rightarrow \infty} c(t)}{\lim _{t \rightarrow \infty} a(t)} .
$$

This and $u=1 / w$ gives the desired result.

3. The asymptotic behavior when $\gamma+b \varepsilon \rho>0$. In this case infectives contribute to susceptibles either by recovery or by births. The endemic equilibrium values of $I$ are given by

$$
I_{N}^{*}=1-\frac{\gamma+b \varepsilon \rho}{\lambda(N)-\alpha_{1}}
$$

where $N$ is $0, N_{0}$ or $\infty$. The asymptotic behavior of $S, I$ and $N$ are analyzed in this section for all possible parameter value regions defined by $r, \alpha_{1}, \theta_{0}, \theta_{\infty}, \sigma_{\infty}, \psi_{0}$ and $\psi_{\infty}$. For example, Theorems $3.4,3.5$ and 3.6 state that if $\theta_{\infty}>1$, then the disease persists in proportion while the population size approaches infinity, or declines to zero, or tends to a finite size. The main results of this section are summarized in Table 1.

\begin{tabular}{|c|c|c|c|c|c|}
\hline & & & $(S, I) \rightarrow$ & $N \rightarrow$ & $(X, Y) \rightarrow$ \\
\hline \multirow[t]{2}{*}{$r<0$} & \multicolumn{2}{|l|}{$\theta_{0} \leq 1$} & $(1,0)$ & 0 & $(0,0)$ \\
\hline & \multicolumn{2}{|l|}{$\theta_{0}>1$} & $\left(1-I_{0}^{*}, I_{0}^{*}\right)$ & 0 & $(0,0)$ \\
\hline \multirow{2}{*}{$\begin{array}{l}r=0 \\
\alpha_{1}=0\end{array}$} & \multicolumn{2}{|l|}{$\theta_{N_{0}} \leq 1$} & $(1,0)$ & $N=N_{0}$ & $\left(N_{0}, 0\right)$ \\
\hline & \multicolumn{2}{|l|}{$\theta_{N_{0}}>1$} & $\left(1-I_{N_{0}}^{*}, I_{N_{0}}^{*}\right)$ & $N=N_{0}$ & $\left(X_{0}, Y_{0}\right)$ \\
\hline \multirow{3}{*}{$\begin{array}{l}r=0 \\
\alpha_{1}>0\end{array}$} & \multicolumn{2}{|l|}{$\theta_{0} \leq 1$} & $(1,0)$ & $N_{e}$ & $\left(X_{e}, 0\right)$ \\
\hline & \multirow{2}{*}{\multicolumn{2}{|c|}{$\theta_{0}>1$}} & & & \\
\hline & & & $\left(1-I_{0}^{*}, I_{0}^{*}\right)$ & 0 & $(0,0)$ \\
\hline \multirow[t]{6}{*}{$r>0$} & \multirow[t]{3}{*}{$\theta_{\infty} \leq 1$} & $\sigma_{\infty}<1$ & $(1,0)$ & $\infty$ & $(\infty, 0)$ \\
\hline & & $\sigma_{\infty}=1$ & $(1,0)$ & $\infty$ & $\left(\infty, Y_{e}\right)$ \\
\hline & & $\sigma_{\infty}>1$ & $(1,0)$ & $\infty$ & $(\infty, \infty)$ \\
\hline & \multirow[t]{3}{*}{$\theta_{\infty}>1$} & $\psi_{\infty} \geq 1$ & $\left(1-I_{\infty}^{*}, I_{\infty}^{*}\right)$ & $\infty$ & $\left(\frac{\gamma+b \varepsilon \rho}{\lim _{N \rightarrow \infty} \lambda(N) / N}, \infty\right)$ \\
\hline & & $\psi_{0}>1>\psi_{\infty}$ & $\left(1-r / \alpha_{1}, r / \alpha_{1}\right)$ & $N_{e}^{*}$ & $\left(X_{e}, Y_{e}\right)$ \\
\hline & & $\psi_{0} \leq 1$ & $\left(1-I_{0}^{*}, I_{0}^{*}\right)$ & 0 & $(0,0)$ \\
\hline
\end{tabular}

TABLE 1. Stability results with $\gamma+b \varepsilon \rho>0$. 
3.1. The case with nonpositive net growth rate constant $(r=b-d \leq$ $0)$.

Theorem 3.1. If $r=b-d<0$, then $X, Y, N \rightarrow 0$ as $t \rightarrow \infty$. If $\theta_{0} \leq 1$ or $I(0)=0$, then $(S, I) \rightarrow(1,0)$ and $N(t)$ decays asymptotically exponentially with rate constant $r$. If $\theta_{0}>1$ and $I(0)>0$, then $(S, I) \rightarrow\left(1-I_{0}^{*}, I_{0}^{*}\right)$ and $N(t)$ decays asymptotically exponentially with rate constant $r-\alpha_{1} I_{0}^{*}$.

Proof. Since $N^{\prime} \leq r N, N(t) \rightarrow 0$ and $(X, Y) \rightarrow(0,0)$. For $\theta_{0}<1$, $\lambda(0)-\left(\alpha_{1}+\gamma+b \varepsilon \rho\right)<0$ so that $(2.2)$ implies that

$$
I^{\prime} \leq-(\gamma+b \varepsilon \rho) I^{2}
$$

for large $t$ so that $I(t) \rightarrow 0$ as $t \rightarrow \infty$. If $I(0)=0$, then $I(t)=0$ and $N(t)=N(0) e^{r t} \rightarrow 0$ as $t \rightarrow \infty$.

For $\theta_{0} \geq 1$ and $I(0)>0$, use Lemma 2.1 (ii) and (iii) to show that $I(t) \rightarrow 0, t \rightarrow \infty$, if $\theta_{0}=1$ and $I(t) \rightarrow I_{0}^{*}, t \rightarrow \infty$, if $\theta_{0}>1$, respectively. The asymptotic behavior of $N(t)$ in the cases above is found by examining $N^{\prime}(t) / N(t)=r-\alpha_{1} I$ as $t \rightarrow \infty$.

From Theorem 3.1, we see that, even in a decreasing population, the disease can still persist proportionately which makes the population decay more quickly to zero.

Theorem 3.2. Let $r=b-d=0$.

1. If $\alpha_{1}=\alpha+b(1-\varepsilon)=0$, then $N(t)=N(0)=N_{0}$ and

$$
(S, I) \rightarrow \begin{cases}(1,0) & \text { if } \theta_{N_{0}} \leq 1 \text { or } I(0)=0 \\ \left(1-I_{N_{0}}^{*}, I_{N_{0}}^{*}\right) & \text { if } \theta_{N_{0}}>1 \text { and } I(0)>0 .\end{cases}
$$

2. If $\alpha_{1}>0$, then $N(t)$ decreases to a finite number $N(\infty)$ with $N(\infty)=0$ or $N(\infty)>0$ and $\theta_{N(\infty)} \leq 1$. Also

$$
(S, I) \rightarrow \begin{cases}(1,0) & \text { if } \theta_{0} \leq 1 \text { or } I(0)=0 \\ \left.1-I_{0}^{*}, I_{0}^{*}\right) & \text { if } \theta_{0}>1 \text { and } I(0)>0 .\end{cases}
$$


Furthermore, $N(\infty)=0$ if $\theta_{0}>1$.

Proof. The proof of 1 is similar to the proof of Theorem 3.1. Consider 2 with $\alpha_{1}>0$. Since $N^{\prime}=-\alpha_{1} I N \leq 0, \lim _{t \rightarrow \infty} N(t)=N(\infty)$ exists and is finite. Thus $\lim _{t \rightarrow \infty} I(t)=I(\infty)$ exists by Lemma 2.1 (if $\theta_{N(\infty)} \geq 1$ ) and by (3.2) if $\theta_{N(\infty)}<1$. Now $0=\lim _{t \rightarrow \infty} N^{\prime}(t)=$ $-\alpha_{1} Y(\infty)$, so $0=Y(\infty)=I(\infty) N(\infty)$. Thus $I(\infty)$ and $N(\infty)$ cannot both be positive. If $N(\infty)>0$, then $I(\infty)=0$, we must have $\theta_{N(\infty)} \leq 1$ since $\theta_{N(\infty)}>1$ implies $I(\infty)>0$ by Lemma 2.1. If $N(\infty)=0$ and $\theta_{0} \leq 1$, then $I(\infty)=0$ by Lemma 2.1 (if $\theta_{0}=1$ ) and by (3.2) (if $\theta_{0}<1$ ). On the other hand, if $\theta_{0}>1$, then $\theta_{N(\infty)}>1$ so $I(\infty)=I_{N(\infty)}^{*}>0$ by Lemma 2.1 and this implies $N(\infty)=0$.

3.2. The cases with positive net growth rate constant $(r=b-d>0)$. With positive net growth rate constant $r$, the disease free population would grow exponentially. In this section we see how the persistence of the disease can change or reverse this growth. The theorem below states that if $\theta_{\infty} \leq 1$, then the disease always dies out proportionately. But since the population size increases to infinity, the number $Y$ of infectives may still grow to infinity. The asymptotic behavior of $Y(t)$ depends on the modified contact number $\sigma_{N}$ defined by (2.4). Note that $\sigma_{N}>\theta_{N}$ for all $N>0$ since $b>d$. Also, $\theta_{N}$ is nondecreasing on $N$, so $\theta_{N} \leq 1$ if $\theta_{\infty} \leq 1$. Thus, for any population size, $\theta_{N}$ is always below the threshold 1 if $\theta_{\infty} \leq 1$ and the disease must be always dying out (in proportion).

Theorem 3.3. If $\theta_{\infty} \leq 1$, then $(S, I) \rightarrow(1,0)$ and $N(t)$ grows exponentially with rate constant $r=b-d$. The number $X(t)$ of susceptibles always approaches $\infty$, but the number $Y(t)$ of infectives approaches to $0, Y_{e}$, or $\infty$ if $\sigma_{\infty}$ is less than, equal to or greater than 1 , respectively.

Proof. Since $\lambda(N)$ is nondecreasing, $\theta_{\infty} \leq 1$ if and only if $\lambda(N)-$ $\left(\alpha_{1}+\gamma+b \varepsilon \rho\right) \leq 0$ for $N \geq 0$. Then (2.2) implies $I^{\prime} \leq-(\gamma+b \varepsilon \rho) I^{2}$ so that $I(t) \rightarrow 0$ as $t \rightarrow \infty$. Hence $N^{\prime}(t) / N \rightarrow r$ and $N \rightarrow \infty$ if $N_{0}>0$.

Since $X=N S, X \rightarrow \infty$ as $t \rightarrow \infty$. If $\sigma_{\infty}<1$, then $\tau=$ $\lambda(\infty)+b \varepsilon(1-\rho)-(\gamma+\alpha+d)<0$. Thus $(2.1)$ implies that $Y^{\prime}(t) \leq \tau Y$ so 
$Y(t) \rightarrow 0$ as $t \rightarrow \infty$. If $\sigma_{\infty}=1$, since $\lambda(N)$ is nondecreasing, we must have $Y^{\prime}(t) \leq 0$ and $Y(t)$ is nonincreasing so that $\lim _{t \rightarrow \infty} Y(t)=Y_{e}$ exists and is finite. If $\sigma_{\infty}>1$, then there exists a positive constant $\delta$ such that $\lambda(N)+b \varepsilon(1-\rho)-(\gamma+\alpha+d) \geq \delta$ for large $t$. Then $Y^{\prime}(t) \geq \delta Y$ for large $t$ which implies $Y(t) \rightarrow \infty$ as $t \rightarrow \infty$.

For the rest of this section, we assume that $\lambda(N)$ is strictly increasing, $\theta_{\infty}>1, I(0)>0$, and $N(0)>0$.

Theorem 3.4. If $\theta_{\infty}>1$ and $\psi_{\infty} \geq 1$, then $(S, I) \rightarrow\left(1-I_{\infty}^{*}, I_{\infty}^{*}\right)$, $N(t)$ increases to infinity and

$$
(X, Y) \rightarrow\left(\frac{b \varepsilon \rho+\gamma}{\lim _{N \rightarrow \infty} \frac{\lambda(N)}{N}}, \infty\right)
$$

as $t \rightarrow \infty$.

Proof. We first show the existence of $N(\infty)=\lim _{t \rightarrow \infty} N(t)$. For $r \geq \alpha_{1}, N^{\prime}(t) \geq\left(r-\alpha_{1}\right) N \geq 0$ so that the conclusion is clear. For $r<\alpha_{1}$, the condition $\psi_{\infty} \geq 1$ implies that $\psi_{N} \geq 1$ for all $N$. In this case, by (2.2), we have $I^{\prime}(t)<0$ at $I=r / \alpha_{1}$, so either $I \geq r / \alpha_{1}$ for all $t$ or $I<r / \alpha_{1}$ for large $t$, and this implies further that either $N^{\prime}(t) \leq 0$ or $N^{\prime}(t)>0$ for large $t$ (see (2.2)). Therefore, $N(\infty)$ exists.

$N(\infty)$ cannot be finite. This is clear for $r>\alpha_{1}$. Suppose for $r \leq \alpha_{1}$ that $N(\infty)$ is finite, then $I(\infty)=0$ if $\theta_{N(\infty)} \leq 1$ and if $\theta_{N(\infty)}>1$

$$
I \rightarrow I_{N(\infty)}^{*}<1-\frac{\gamma+b \varepsilon \rho}{\lambda(\infty)-\alpha_{1}} \leq \frac{r}{\alpha_{1}} .
$$

In both cases, we have $(d N / N) / N \rightarrow r-\alpha_{1} I(\infty)>0$, a contradiction. So $N(\infty)=\infty$ and $I \rightarrow I_{\infty}^{*}$ by Lemma 2.1.

Since $I_{\infty}^{*}>0, Y(t)=N(t) I(t) \rightarrow \infty$ as $t \rightarrow \infty$. Solving for $X$ in terms of $Y(t)$ and $N(t)$ from the first equation of (2.1) and using L'Hopital's rule gives

$$
\lim _{t \rightarrow \infty} X(t)=\frac{b \varepsilon \rho+\gamma}{\lim _{t \rightarrow \infty} \frac{\lambda(N)}{N}}
$$


We give a few remarks on Theorem 3.4. Since $\psi_{N}$ is a strictly decreasing function of $N, \psi_{\infty} \geq 1$ implies that $\psi_{N}>1$ for all $N$, and this means that the population size always increases to infinity. If $\psi_{\infty}>1$, then $I_{\infty}^{*}<r / \alpha_{1}$, and the population size increases exponentially with the reduced rate constant $r-\alpha_{1} I_{\infty}^{*}$. If $\psi_{\infty}=1$, then although the population size still increases to infinity, it may not increase in an exponential way. Thus, the disease has a strong effect on the growth rate of the host population.

Theorem 3.5. If $\theta_{\infty}>1$ and $\psi_{0} \leq 1$, then the population dies out and $(S, I) \rightarrow\left(1-I_{0}^{*}, I_{0}^{*}\right)$ as $t \rightarrow \infty$.

Proof. Now $\psi_{0} \leq 1$ implies that $I^{\prime}>0$ if $I=r / \alpha_{1}$ and $N>0$. A similar argument as in the proof of Theorem 3.4 shows the existence of $N(\infty)=\lim _{t \rightarrow \infty} N(t)$. Note that $\psi_{0} \leq 1$ implies that $\theta_{N}>0$ for all $N \geq 0$ so that $I(t) \rightarrow I_{N(\infty)}^{*}$ by Lemma 2.1 ; note also that $I_{N}^{*}>r / \alpha_{1}$ for $N>0$. Suppose $N(\infty)>0$, then $I_{N(\infty)}^{*}>r / \alpha_{1}$, and $N^{\prime}(t) / N$ has limit $r-\alpha_{1} I_{N(\infty)}^{*}<0$ as $t \rightarrow \infty$, which is a contradiction. Hence, $\lim _{t \rightarrow \infty} N(t)=0$, and the results follow.

The theorem above says that if $\psi_{0} \leq 1$, then the population always dies out and the disease persists in proportion but not in number. If $\psi_{0}<1$, then $I_{0}^{*}>r / \alpha_{1}$ so the population size decreases exponentially with rate constant $r-\alpha_{1} I_{0}^{*}$. If $\psi_{0}=1$, then the population size also declines to zero, but may not decline exponentially.

Theorem 3.6. If $\theta_{\infty}>1$ and $\psi_{\infty}<1<\psi_{0}$, then $N(t) \rightarrow N_{e}^{*}$ where $N_{e}^{*}>0$ satisfies

$$
\lambda\left(N_{e}^{*}\right)=\frac{\alpha+d+\gamma-b \varepsilon(1-\rho)}{1-r / \alpha_{1}}
$$

and $(S, I) \rightarrow\left(1-r / \alpha_{1}, r / \alpha_{1}\right)$ as $t \rightarrow \infty$.

Proof. We need only to show that $I \rightarrow r / \alpha_{1}$ and $N \rightarrow N_{e}^{*}$. Using (2.2) and the given condition $\psi_{0}>1>\psi_{\infty}$, one can easily check that (2.2) has a unique positive equilibrium point $\left(r / \alpha_{1}, N_{e}^{*}\right)$ in $D$. To show 
global asymptotic stability, construct the Lyapunov function

$$
V(I, N)=\int_{r / \alpha_{1}}^{I} \frac{\alpha_{1} I-r}{I(1-I)} d I+\int_{N_{e}^{*}}^{N} \frac{\lambda(N)-\lambda\left(N_{e}^{*}\right)}{N} d N .
$$

It is clear that $V(I, N)$ is positive definite in $D^{0}$ (the interior of $D$ ) with $V=0$ if and only if $N=N_{e}^{*}$ and $I=r / \alpha_{1}$ and $V(I, N) \rightarrow \infty$ if $(I, N)$ approaches the boundary of $D$ (i.e., $I \rightarrow 0$, or $I \rightarrow 1$, or $N \rightarrow 0$, or $N \rightarrow \infty)$. Also

$$
\begin{aligned}
\frac{d V}{d t} & =-\left(r-\alpha_{1} I\right)\left(\lambda\left(N_{e}^{*}\right)-\alpha_{1}-\frac{\gamma+b \varepsilon \rho}{1-I}\right) \\
& =-\frac{(\gamma+b \varepsilon \rho)\left(r-\alpha_{1} I\right)^{2}}{\left(\alpha_{1}-r\right)(1-I)} \leq 0
\end{aligned}
$$

and $d V / d t=0$ if and only if $I=r / \alpha_{1}$. On $\{D \backslash\{I N=0\}\} \cap\left\{I=r / \alpha_{1}\right\}$, the point $\left(r / \alpha_{1}, N_{e}^{*}\right)$ is the largest positive invariant set, and $I^{\prime}<0$ on $I=1$. A Lyapunov theorem in $\left[\mathbf{1 4}\right.$, p. 226] implies that $\left(r / \alpha_{1}, N_{e}^{*}\right)$ must be globally asymptotically stable in $D \backslash\{I N=0\}$.

4. The asymptotic behavior when $\gamma+b \varepsilon \rho=0$. If $\gamma+b \varepsilon \rho=0$, then $\gamma$ must be 0 so that no one recovers and the SIS model reduces to an $S I$ model. Moreover, $b$ or $\varepsilon$ or $\rho$ must be 0 so that either there are no births $(b=0)$ or the infectives have no offspring $(\varepsilon=0)$ or all the newborns of infectives are infected $(\rho=0)$. Note from the transfer diagram that $\gamma+b \varepsilon \rho=0$ implies that there is no feedback from the infective class $Y$ into the susceptible class $X$. In this case the system (2.2) reduces to

$$
\begin{aligned}
I^{\prime}(t) & =\left(\lambda(N)-\alpha_{1}\right) I(1-I) \\
N^{\prime}(t) & =N\left(r-\alpha_{1} I\right) .
\end{aligned}
$$

This system is simpler than (2.2), but there are more possible cases in the asymptotic behavior. Assume that $\lambda(N)$ is strictly increasing. Since $\theta_{N}, \sigma_{N}$ and $\psi_{N}$ simplify greatly when $\gamma+b \varepsilon \rho=0$, the results in Table 2 are given in terms of values of $\lambda(N), r$ and $\alpha_{1}$ instead of using $\theta_{N}, \sigma_{N}$ and $\psi_{N}$ as in Table 1 . One can easily check that $\theta_{N} \leq 1$ if and only if $\lambda(N) \leq \alpha_{1}, \sigma_{N} \leq 1$ if and only if $\lambda(N)-\alpha_{1}+r \leq 0$. Moreover, 
if $\theta_{\infty}>1$, then $\psi_{\infty} \geq 1$ if and only if $r \geq \alpha_{1}, \psi_{0} \leq 1$ and $r \neq \alpha_{1}$ if and only if $r<\alpha_{1}$ and $\lambda(0) \geq \alpha_{1}, \psi_{0}>1>\psi_{\infty}$ if and only if $\lambda(0)<\alpha_{1}$ and $r<\alpha_{1}$. For most functions $\lambda(N)$ seen in the literature, $\lambda(0)=0$ so that many of the cases in Table 2 do not occur. Note that $I=0$, $I=1, N=0$ are solutions for (4.1) and we need only to discuss (4.1) in $D^{0}$.

TABLE 2. Stability results with $\gamma+b \varepsilon \rho=0$.

\begin{tabular}{|c|c|c|c|c|c|}
\hline & & & $(S, I) \rightarrow$ & $N \rightarrow$ & $(X, Y) \rightarrow$ \\
\hline \multirow[t]{3}{*}{$r<0$} & $\lambda(0)<\alpha_{1}$ & & $(1,0)$ & 0 & $(0,0)$ \\
\hline & $\lambda(0)=\alpha_{1}$ & & $\left(1-I_{e}, I_{e}\right)$ & 0 & $(0,0)$ \\
\hline & $\lambda(0)>\alpha_{1}$ & & $(0,1)$ & 0 & $(0,0)$ \\
\hline \multicolumn{6}{|l|}{$\alpha_{1}=0$} \\
\hline \multicolumn{5}{|l|}{$\alpha_{1}>0$} & $\left(X_{e}, 0\right)$ \\
\hline & $\lambda(0)=\alpha_{1}$ & & $\left(1-I e, I_{e}\right)$ & 0 & $(0,0)$ \\
\hline & $\lambda(0)>\alpha_{1}$ & & $(0,1)$ & 0 & $(0,0)$ \\
\hline \multirow[t]{11}{*}{$r>0$} & $\lambda(\infty)<\alpha_{1}$ & $\lambda(\infty)<\alpha_{1}-r$ & $(1,0)$ & $\infty$ & $(\infty, 0)$ \\
\hline & & $\lambda(\infty)=\alpha_{1}-r$ & $(1,0)$ & $\infty$ & $\left(\infty, Y_{e}\right)$ \\
\hline & & $\lambda(\infty)>\alpha_{1}-r$ & $(1,0)$ & $\infty$ & $(\infty, \infty)$ \\
\hline & $\lambda(\infty)=\alpha_{1}$ & & $\left(1-I_{e}, I_{e}\right)$ & $\infty$ & $(\infty, \infty)$ \\
\hline & $\lambda(\infty)>\alpha_{1}$ & $\lambda(\infty)>r>\alpha_{1}$ & $(0,1)$ & $\infty$ & $(0, \infty)$ \\
\hline & & $\lambda(\infty)=r$ & $(0,1)$ & $\infty$ & $(X(\infty), \infty)$ \\
\hline & & $\alpha_{1}<\lambda(\infty)<r$ & $(0,1)$ & $\infty$ & $(\infty, \infty)$ \\
\hline & & $r=\alpha_{1}$ & $(0,1)$ & $N(\infty)$ & $(0, Y(\infty))$ \\
\hline & & $\lambda(0)>\alpha_{1}>r$ & $(0,1)$ & 0 & $(0,0)$ \\
\hline & & $\lambda(0)=\alpha_{1}>r$ & $\left(1-I_{e}, I_{e}\right)$ & 0 & $(0,0)$ \\
\hline & & $\lambda(0)<\alpha_{1}>r$ & periodic & periodic & periodic \\
\hline
\end{tabular}

4.1. The special cases with $r=b-d \leq 0$. For $r=b-d<0,(4.1)$ implies that $X, Y, N \rightarrow 0$ as $t \rightarrow \infty$. If $\theta_{0}>1$ so that $\lambda(0)>\alpha_{1}$, then $I^{\prime}(t)>0$ and $I(\infty)=\lim _{t \rightarrow \infty} I(t)$ exists and is positive. From $I^{\prime}(t) \rightarrow 0$ we get $I(\infty)=1$ and $(S, I) \rightarrow(0,1)$ and $N(t)$ decays exponentially with rate constant $r-\alpha_{1}$. If $\theta_{0}<1$, then for large $t, I^{\prime}(t)<0$ in $D^{0},(S, I) \rightarrow(1,0)$ and $N(t)$ decays asymptotically and exponentially with rate constant $r$. If $\theta_{0}=1$ so that $\lambda(0)=\alpha_{1}$, and 
$I^{\prime}(t)>0$ in $D^{0}$, then $I(\infty)$ exists and $(S, I) \rightarrow(1-I(\infty), I(\infty))$. Note that for $\theta_{0}=1$, the line of equilibria on $\{N=0\} \cap D$ are neutrally stable for (4.1).

Consider the case $r=b-d=0$. If $\alpha_{1}=0$, then $N(t)=N(0)=N_{0}$; if $N_{0}>0$, then $(S, I) \rightarrow(0,1)$. If $\alpha_{1}>0$, then $N(\infty)=\lim _{t \rightarrow \infty} N(t)$ exists and is finite. Since $N^{\prime}=-\alpha_{1} Y \rightarrow 0, Y(t) \rightarrow 0$ as $t \rightarrow \infty$. If $\theta_{0}>1$ so that $\lambda(0)>\alpha_{1}$, then $I^{\prime}(t) \geq 0$, and $I(\infty)=\lim _{t \rightarrow \infty} I(t)$ exists and is positive. From $I^{\prime}(t) \rightarrow 0$ we get $I(\infty)=1$ and $(S, I) \rightarrow(0,1)$; furthermore, $N^{\prime}(\infty)=-\alpha_{1} N(\infty)=0$ implies $N(\infty)=0$. If $\theta_{0}=1$ so that $\lambda(0)=\alpha_{1}$ and $I^{\prime}(t)>0$ in $D^{0}$, then $I(\infty)$ exists and $(S, I)$ approaches $(1-I(\infty), I(\infty))$. Since $N^{\prime}(\infty)=-\alpha_{1} N(\infty) I(\infty)=0$ and $\alpha_{1} I(\infty)>0, N(\infty)=0$. If $\theta_{0}<1$ so that $\lambda(0)<\alpha_{1}$, then $\theta_{N(\infty)}<1$ (if $\theta_{N(\infty)} \geq 1$, then $N(\infty)>0$ and $I(t) \geq I(0)>0$ implies $N^{\prime}(\infty)=-\alpha_{1} N(\infty) I(\infty)<0$ and $\left.N(\infty)=-\infty\right)$ and $I(\infty)=0$.

4.2. The cases with $r=b-d>0$. If $\theta_{\infty} \leq 1$, i.e., $\lambda(\infty) \leq \alpha_{1}$, then $I^{\prime}(t)<0$ in $D^{0}$ and $I(\infty)=\lim _{t \rightarrow \infty} I(t)$ exists. Supopse $\alpha_{1} I(\infty) \geq r$, then $\alpha_{1} I(t)>r$ which implies from (4.1) that $N(\infty)<\infty$. Then $\lambda(N(\infty))-\alpha_{1}<0$ so $I(t) \rightarrow 0$ which contradicts $\alpha_{1} I(\infty) \geq r>0$. Hence $\alpha_{1} I(\infty)<r$ and $N(\infty)=\infty$. If $\theta_{\infty}<1$, then $I^{\prime}(t)<0$ implies $I(\infty)=0$ so the disease dies out proportionately. For $\theta_{\infty}=1$, we know that $\alpha_{1} I(\infty)<r$. In all cases, if $\theta_{\infty} \leq 1$, the population still increases exponentially but at a reduced rate if $\theta_{\infty}=1$. How does $\sigma_{\infty}$ determine $Y(\infty)$ ? When $\sigma_{\infty}<1$ so $I \rightarrow 0$ and $N \rightarrow \infty$, checking the equation in $Y^{\prime}(t)$, one finds $Y \rightarrow 0$. If $\sigma_{\infty}>1$, then $Y \rightarrow \infty$. If $\sigma_{\infty}=1$, then $Y^{\prime}(t) \leq 0$ so that $Y(t) \rightarrow Y_{e}$.

Now consider the cases where $\theta_{\infty}>1$ (i.e., $\lambda(\infty)>\alpha_{1}$ ). If $r>\alpha_{1}$, then $N^{\prime}(t) \geq\left(r-\alpha_{1}\right) N$ so $N(t) \rightarrow \infty$. This implies from (4.1) that $I(t) \rightarrow 1$ as $t \rightarrow \infty$. Since $X^{\prime}(t)=(r-\lambda(N) I) X$, the asymptotic behavior of $X$ depends on the sign of $r-\lambda(\infty)$. If $r=\alpha_{1}$, then $N(t)$ is nondecreasing so $N(t) \rightarrow N(\infty)$ with $\lambda(N(\infty))>\alpha_{1}$, and $I(t) \rightarrow 1$. In the case where $r<\alpha_{1}$, if $\lambda(0) \geq \alpha_{1}$, then $I(t)$ is increasing and $I(\infty)$ exists. Suppose $\alpha_{1} I(\infty) \leq r$, then $\alpha_{1} I(t)<r$ which implies from (4.1) that $N(\infty)>0$. Then $\lambda(N(\infty))-\alpha_{1}>0$ so $I(t) \rightarrow 1$ which contradicts $r<\alpha_{1}$. Thus $\alpha_{1} I(\infty)>r$ so $N(\infty)=0$. If $\lambda(0)>\alpha_{1}$, then $I(\infty)=1$ by (4.1). If $\lambda(0)=\alpha_{1}$, then $I(\infty) \in\left(r / \alpha_{1}, 1\right)$ and $N(t)$ decreases exponentially to zero. If $\lambda(0)<\alpha_{1}$, then (4.1) has a unique positive equilibrium point $\left(r / \alpha_{1}, N_{e}\right)$ in $D^{0}$ with $\lambda\left(N_{e}\right)=\alpha_{1}$. Note 
that $V(I, N)=C$ (given by (3.4)) is the integral for (4.1) since $V^{\prime}=0$. Hence $\left(r / \alpha_{1}, N_{e}\right)$ is a center for (4.1) and all solution paths starting in $D^{0}$ are periodic.

The occurrence of a center with periodic solutions for these parameter values is rather surprising since there is no feedback from the infective class $Y$ into the susceptible class $X$.

5. Discussion. The model considered here is an SIS model with exponential births and deaths and with general total population size dependent contact function $\lambda(N)$. Special cases of $\lambda(N)$ have been considered by Anderson and May [1] where $\lambda(N)$ is proportional to $N$, and by Busenberg and Van den Driessche [7], and Busenberg and Hadeler [6] where $\lambda(N)$ is a constant and is independent of total population size. Thieme $[\mathbf{1 8}, \mathbf{1 9}]$ has obtained results similar to ours for an SIRS model with a population size dependent contact rate, but his results are primarily local results since his model is more complicated.

We have found threshold parameters $\theta_{0}, \psi_{0}, \theta_{\infty}, \psi_{\infty}$ and $\sigma_{\infty}$, which are determined simply by $\lambda(0), \lambda(\infty)$ and model parameters. These threshold quantities completely determine the global asymptotic behavior of the epidemic and demographic dynamics. The quantity $\theta_{0}$ determines whether or not the disease persists proportionately in a decreasing population, and the quantity $\theta_{\infty}$ determines whether or not the disease persists proportionately in an increasing population.

When $\theta_{\infty}>1$ so that the disease persists, this disease persistence can affect the natural growth rate of the population and can even reverse this growth. If $\psi_{\infty}>1$, then the population size still grows exponentially, but at a slower rate. If $\psi_{0}>1>\psi_{\infty}$, then the population size approaches an equilibrium value instead of growing exponentially. If $\psi_{0}<1$, then the population size declines exponentially to zero. If $\psi_{\infty}=1$, then the population size still grows to infinity, but may not grow in an exponential way. Also, if $\psi_{0}=1$, then the population size declines to zero which may not be in an exponential way. Thus, the persistence of the disease can have a strong impact on the behavior of the population size.

Some aspects of this model are very interesting. First, no matter how complicated the contact process is, the contact function $\lambda(N)$ for a small population size and large population size determine the 
endemic and demographic dynamics. Second, in decreasing population, the infectious proportion may tend to a positive endemic equilibrium $I_{0}^{*}$, while the total number of infectives is tending to zero. On the other hand, in an increasing population, the fraction of the infectives may tend to zero while the number of infectives is tending to a finite value or infinity (see the case $\theta_{\infty} \leq 1, \sigma_{\infty} \geq 1$ ). Third, in the case where there is no recovery and no newborns are susceptibles, there is an endemic center for some parameter values. This is the first time known to us when a center has occurred in an epidemiological model.

Acknowledgment. The author thanks Professor H.W. Hethcote for constructive comments and discussions concerning this material.

\section{REFERENCES}

1. R.M. Anderson and R.M. May, Population biology of infectious diseases, Part I. Nature 280 (1979), 361-367.

2. R.M. Anderson, Transmission dynamics and control of infectious diseases, in Population biology of infectious diseases (R.M. Anderson, R.M. May, eds.), 149-176, Life Sciences Research Report 25, Dahlem Conference, Berlin 1982, Springer.

3. V. Andreason, Disease regulation of age-structured host populations, Theoret. Population Biol. 36 (1989), 214-239.

4. F. Brauer, Models for the spread of universally fatal diseases, J. Math. Biol. 28 (1990), 451-462.

5. F. Brauer, Models for the spread of universally fatal diseases, II, in Differential equations, models in biology, epidemiology, and ecology (S. Busenberg and M. Martelli, eds.), Lecture Notes in Biomath. 92 (1991) Springer-Verlag, Berlin, Heidelberg, New York, 57-69.

6. S. Busenberg and K.P. Hadeler, Demography and epidemics, Math. Biosci. 101 (1990), 63-74.

7. S. Busenberg and P. Van den Driessche, Analysis of a disease transmission model in a population with varying population size, J. Math. Biol. 29 (1990), $257-270$.

8. C. Castillo-Chavez, K.L. Cooke, W. Huang and S.A. Levin, On the role of long incubation periods in the dynamics of acquired immunodeficiency syndrome (AIDS), Part 1, Single populuation models, J. Math. Biol. 17 (1989), 373-398.

9. - On the role of long incubation periods in the dynamics of acquired immunodeficiency syndrome (AIDS), Part 2, Multiple group models, in Mathematical and statistical approaches to AIDS epidemiology (C. Castillo-Chavez, ed.), Lecture Notes in Biomath. 83 (1989), Springer-Verlag, 200-217.

10. L.Q. Gao and H.W. Hethcote, Diseases transmission models with densitydependent demographics, J. Math. Biol. 30 (1992), 717-731. 
11. J.A.P. Heesterbeek and J.A.J. Metz, The saturating contact rate in marriage and epidemic models, J. Math. Biol. 31 (1993), 529-539.

12. C.S. Holling, The functional response of invertebrate predators to prey density, Mem. Ent. Soc. Canada 48 (1966).

13. J. Mena-Lorca and H.W. Hethcote, Dynamic models of infectious diseases as regulators of population sizes, J. Math. Biol. 30 (1992), 693-716.

14. R.K. Miller and A.N. Michel, Ordinary differential equations, New York, Academic Press, 1982.

15. A. Pugliese, Population models for diseases with no recovery, J. Math. Biol. 28 (1990), 65-82.

16. - An $S \rightarrow E \rightarrow I$ epidemic model with varying population size, in Differential equation models in biology, epidemiology, and ecology (S. Busenberg and M. Marteli, eds.). Lecture Notes in Biomath. 92 (1991), Springer-Verlag, Berlin, Heidelberg, New York, 121-138 .

17. H.R. Thieme and C. Castillo-Chavez, How many infection-age dependent infectivity affect the dynamics of HIV/AIDS?, preprint, 1992.

18. H.R. Thieme, Persistence under relaxed point-dissipativity, (with application to an endemic model), SIAM J. Math. Anal. 24 (1993), 407-435.

19. - Epidemic and demographic interaction in the spread of potentially fatal diseases in growing populations, Math. Biosci. 111 (1992), 99-130.

Department of Mathematics, The University of Iowa, Iowa City, iA 52242 